\title{
Basins of Attraction of a Nonlinear Nanomechanical Resonator
}

\author{
I. Kozinsky, ${ }^{*}$ H. W. Ch. Postma, ${ }^{\dagger}$ O. Kogan, A. Husain, ${ }^{\ddagger}$ and M. L. Roukes ${ }^{\S}$ \\ Kavli Nanoscience Institute and Condensed Matter Physics 114-36, California Institute of Technology, \\ Pasadena, California 91125, USA
}

(Received 26 May 2007; published 13 November 2007)

\begin{abstract}
We present an experiment that systematically probes the basins of attraction of two fixed points of a nonlinear nanomechanical resonator and maps them out with high resolution. We observe a separatrix which progressively alters shape for varying drive strength and changes the relative areas of the two basins of attraction. The observed separatrix is blurred due to ambient fluctuations, including residual noise in the drive system, which cause uncertainty in the preparation of an initial state close to the separatrix. We find a good agreement between the experimentally mapped and theoretically calculated basins of attraction.
\end{abstract}

DOI: 10.1103/PhysRevLett.99.207201

PACS numbers: $85.85 .+\mathrm{j}, 05.45 .-\mathrm{a}$

In the last few years the dimensions of mechanical devices have been scaled deep into the submicrometer regime, which resulted in the increased detection sensitivity of extremely small physical quantities $[1,2]$ and in an enhancement of the significance of nonlinear dynamics in such devices [3]. Deliberately operating the system in the nonlinear regime can improve precision of some experimental measurements on nanoscale. For example, a nonlinear resonator can be employed to suppress amplifier noise in an oscillator circuit [4], noise-induced switching between two stable states in a nonlinear beam resonator enables precision measurement of the resonant frequency [5], and the sensitivity of a resonator for mass detection can be improved when the resonator is driven into a region of nonlinear oscillations [6]. Finally, in a Josephson junction, which is dynamically similar to a mechanical resonator in the nonlinear regime, the bistable state of the nonlinear system can be used as a bifurcation amplifier to perform a nondissipative, low-backaction measurement of the phase across the junction [7]. When nanomechanical devices reach the quantum-limited regime [8], a nanomechanical version of such an amplifier could be used for a similar sensitive low-back-action measurement of the state of a quantum mechanical resonator. The nonlinear response of nanomechanical resonators could also be used to detect transition from the classical to quantum regime $[9,10]$. It is therefore important to understand nonlinear dynamics of these systems well, so that we can fully realize their potential in expanding our experimental capabilities.

Although some work has been done with parametric systems $[11,12]$, the majority of nonlinear nanoscale systems that have been studied are directly driven $[5,13,14]$ and the dominant nonlinearity in the restoring force is cubic, also known as Duffing nonlinearity. When a system is driven strongly, the Duffing nonlinearity causes the resonance response curve to become asymmetric. When the resonance is pulled far enough to one side, hysteretic behavior is observed as two stable states appear in the system [15]. The stable states, known as "attractors" or "fixed points", correspond to the points in state space to which the system converges with time. For each attractor, a set of initial states that dynamically evolves to that attractor forms its basin of attraction, which is separated from the rest of the state space by the separatrix curve.

There have been very few experimental studies of basins of attraction because following the evolution of initial conditions in low-frequency macroscopic systems is usually very time consuming and system parameters tend to drift over the course of many data-taking runs. Previous mappings of basins of attraction $[16,17]$ used the method of stochastic interrogation, where the system is stochastically perturbed and initial states are sampled at random without fully covering the basins.

We demonstrate the ability to systematically prepare a nonlinear, Duffing-type nanomechanical resonator in the required set of initial states and map the basins of attraction of its two fixed points with high resolution. The fact that the relevant time scale per data point, $\sim Q / f_{0}$, is very short for very-high-frequency nanometer-scale devices, allows us to take many data points in a relatively short time with minimal drift in the parameters of the system. We also observe that the separatrix changes shape for varying drive strength, so that one of the basins becomes progressively smaller and eventually disappears.

The device used for mapping the basins of attraction, a doubly-clamped platinum nanowire, is shown in the scanning electron microscope (SEM) photograph in Fig. 1(a). The nanowire, with a length $L$ of $2.25 \mu \mathrm{m}$ and a diameter of $35 \mathrm{~nm}$, is grown by electrodeposition of platinum into a nanoporous membrane [18]. Gold contact pads on both ends and a gate are fabricated using electron beam lithography, and about $150 \mathrm{~nm}$ of the substrate is subsequently etched away in hydrofluoric acid to suspend the device [13]. We actuate and detect the vibration of the nanowire magnetomotively [19] in a magnetic field, $B=8 \mathrm{~T}$, in a cooled (to about $20 \mathrm{~K}$ ) probe in vacuum. The magnetic field is applied perpendicular to the device so that the vibration is in the plane of the gate electrode. At low 

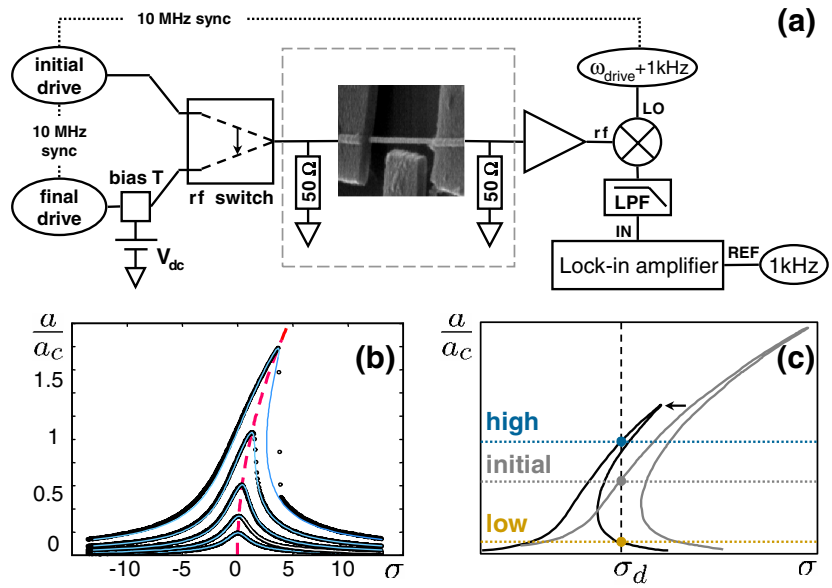

FIG. 1 (color online). (a) Experimental layout. The initial drive prepares an initial state of the platinum nanowire resonator (shown in the SEM photo), a $5 \mathrm{~ns}$ rf switch is then flipped to connect to the final drive, and the state of the device is measured by a lock-in amplifier after mixing down to a low $(1 \mathrm{kHz})$ frequency and low-pass filtering (LPF) the residual rf signal. (b) Vibration amplitude versus frequency, for various driving powers at the sample $(-90,-85,-80,-75,-70 \mathrm{dBm}$, or normalized to the critical drive $V_{c}=28.4 \mu \mathrm{V}, V / V_{c}=0.249$, $0.443,0.788,1.401,2.492)$ showing the onset of nonlinearity in the platinum nanowire resonator. (c) The state of the nanowire resonator is first prepared in an initial state on the initial response curve (gray) by choosing an appropriate drive strength for the fixed detuning frequency, $\sigma_{d}=4$.26. After the $\mathrm{rf}$ switch is flipped to connect the final drive, the response curve changes to the one shown in black and the initial state evolves to either the high-amplitude state or low-amplitude state. The small bias voltage, applied to the nanowire together with the final drive, is chosen so that the hysteresis loop of the initial curve is at higher frequency than the operating frequency.

driving powers the resonance curve is linear, and we extract a resonant frequency of $45.35 \mathrm{MHz}$ and mechanical quality factor of 6045 . The resonant frequency is higher than the expected $17.71 \mathrm{MHz}$ for this device geometry, most likely due to differential thermal contraction between the silicon wafer and the gold contacts that results in residual tension. The ratio of electromechanical impedance, $R_{\mathrm{em}}$, to electrical impedance, $R_{e}$, is 0.222 , which indicates the presence of significant eddy current damping [20]. The quality factor corrected for the eddy current damping is $Q_{0}=Q /\left(1-R_{\mathrm{em}} / R_{e}\right)=7770$.

The resonant response of the nanowire to different drives is shown in Fig. 1(b). The nonlinearity of the device is fully characterized by the critical amplitude $a_{c}$, the point where the resonance curve develops infinite slope. The theoretical curves (thin solid lines) in Fig. 1(b) are generated using the critical amplitude, $a_{c}$, as the only fitting parameter. We determine $a_{c}$ by fitting the backbone curve that connects the peaks of resonant curves for different drives to the theoretical expression $\left(a_{p} / a_{c}\right)^{2}=\sqrt{3} \sigma / 2$ [21], where $\sigma \equiv 2 Q\left(f / f_{0}-1\right)$ is the detuning frequency scaled by the width of the resonance and $a_{p}$ is the peak amplitude. The extracted critical amplitude $a_{c}$ is $2.68 \mathrm{~nm}$.

The dominant source of nonlinearity in doubly clamped nanomechanical resonators is the additional tension in the beam that appears when vibrations are sufficiently large. This extra tension gives rise to a cubic nonlinearity in the spring constant term in the equation of motion [3], giving it a Duffing oscillator form:

$$
\ddot{x}+\frac{\omega_{0}}{Q} \dot{x}+\omega_{0}^{2}\left(x+\alpha x^{3}\right)=F \cos (\Omega t) .
$$

Here, $x(t)$ is the displacement of the beam, $\omega_{0}=2 \pi f_{0}$ is the resonance frequency, $\alpha=2 \sqrt{3} /\left(9 a_{c}^{2} Q\right)$ is the nonlinearity parameter [21], $\Omega=2 \pi f=(\sigma /(2 Q)+1) \omega_{0}$ is the driving frequency, and $F$ is the force per unit mass acting on the resonator of mass $m$. The driving force in the magnetomotive transduction is the Lorentz force that acts on the nanowire when a current $I_{d}(t)$ is passed through it in a magnetic field, $F=L B I_{d}(t) / \mathrm{m}$. When the driving voltage amplitude is $V_{d}$, the driving current is given by $I_{d} \approx$ $V_{d} / R_{e}$

As illustrated in Fig. 1(a), we prepare the system by exciting it with an initial-drive amplitude, $V_{i}$, and then switching to a final drive amplitude, $V_{f}$. Two rf sources (HP 8648B for initial and SR DS345 with a frequency doubler for final drive) are tuned to the same fixed frequency off resonance, $\sigma_{d}=4.26$. Their internal clocks and that of the local oscillator (LO) are synchronized with their $10 \mathrm{MHz}$ clock reference. By changing the phase difference $\phi$ between final and initial drives while holding the initialdrive amplitude, $V_{i}$, constant, we can prepare the resonator in the initial states corresponding to a circle in state space. By also stepping the initial-drive values, $V_{i}$, we can cover a disk of initial states in state space. We switch rapidly from the initial to final drive using a $5 \mathrm{~ns}\left(<1 / f_{0} \sim 22 \mathrm{~ns}\right) \mathrm{rf}$ switch (Mini-Circuits ZASWA-2-50DR). After the switching occurs, we measure the final amplitude of the oscillator and mark it as a low or a high final amplitude. In order to access a continuum of initial states, we apply a small dc bias voltage of $V_{\mathrm{dc}} \approx 10 \mathrm{mV}$ to the wire in the final state. The capacitive interaction with the gate lowers the resonant frequency of the final state [22] so that the hysteretic frequency response of the final state occurs at the same frequency as the single-valued resonant response of the initial state [Fig. 1(c)]. Without this technique, the resonator cannot be prepared in the initial states corresponding to the unstable branch of the initial-drive resonance and an annulus of these states in state space would not be accessible in the experiment.

To map the basins of attraction in this manner, the initial states were driven at -90 to $-50 \mathrm{dBm}$ in 60 concentric circles with 60 phase points per circle, corresponding to a displacement range of 0 to $2.5 a_{c}$, where $a_{c}$ is the critical amplitude extracted from experimental data [Fig. 1(b)]. Each of the initial states was marked according to the attractor that it evolved to after the switch was flipped: 
blue for the high-amplitude state and yellow for the lowamplitude state. These data were rerastered using a nearest neighbor search algorithm to create the continuous color plots shown in Fig. 2.

For very low final drives, there is only one state the resonator can occupy [refer to Fig. 1(b)]. As the final drive starts exciting a nonlinear response, a second basin representing the high-amplitude stable state appears, but subtends a smaller fraction of the state space [Fig. 2(1)]. When the final drive amplitude is increased, the high-amplitude basin grows, and the low-amplitude basin gets progressively smaller and eventually disappears [Fig. 2(6)]. The disappearance of the low-amplitude state can also be observed in Fig. 1(b): a large final drive results in a wider hysteretic region that moves to the right, where the lowamplitude branch starts at $\sigma>\sigma_{d}$, so only the highamplitude state is available to the system.

To calculate the location of the fixed points and the separatrix from Eq. (1), we separate the fast dynamics on a time scale of $1 / \omega_{0}$, from the slow dynamics on a longer time scale $Q / \omega_{0}$, associated with a detuning $\sigma$ from resonance as well as damping and nonlinearity [21]. The solution to Eq. (1) can be written as $x_{0}(t, T)=A(T) e^{i \omega_{0} t}+$ $\bar{A}(T) e^{-i \omega_{0} t}$, where $t$ characterizes the fast dynamics and $T$, the slow dynamics. The slowly-varying amplitude $A(T)=$ $[X(T)+i Y(T)] \exp \left(i \omega_{0} \sigma / 2 T\right)$ obeys the envelope equations:

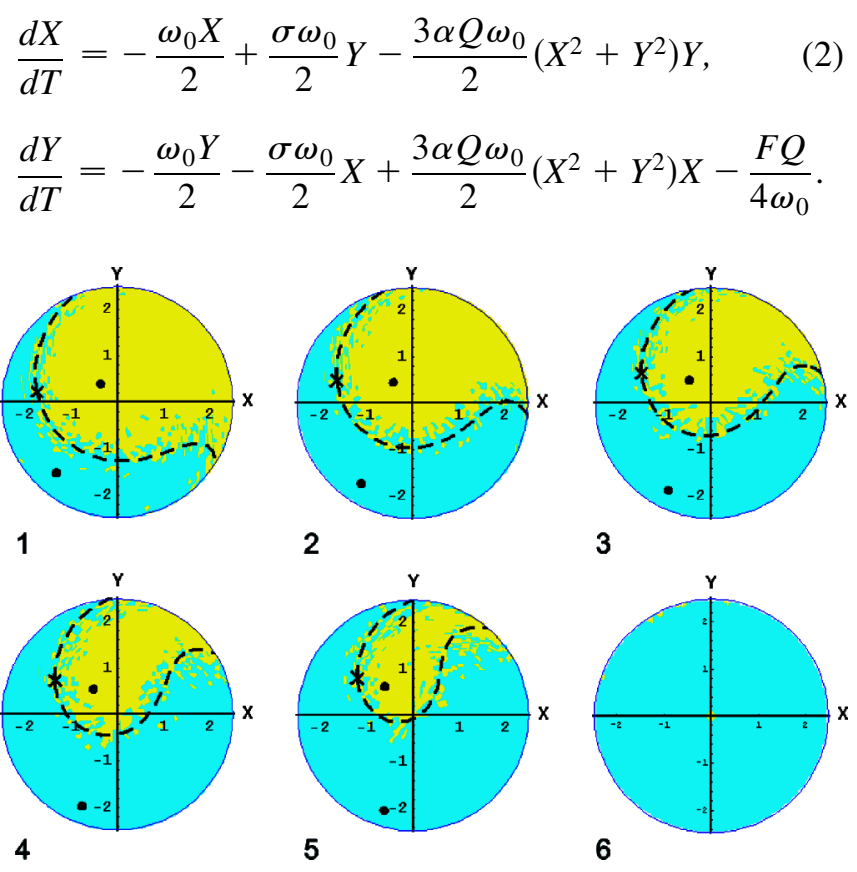

FIG. 2 (color online). Basins of attraction of a nanowire resonator at a fixed frequency, $\sigma_{d}=4.26$, for increasing final drive values, $\quad V_{f} / V_{c}=(1) 1.867, \quad$ (2) $2.049, \quad$ (3) $2.237, \quad$ (4) 2.434 , (5) 2.640 , (6)2.741. Blue and yellow colors indicate the final high- and low-amplitude states, respectively. The data consist of 60 concentric circles with 60 points each, corresponding to a displacement range of 0 to $2.5 a_{c}$. Theoretical fixed points, saddle point, and separatrix curve are indicated by black points, black cross, and the dashed black curve, respectively.
We have assumed here that $1 / Q \ll 1$, and the slowlyvarying amplitude approximation implies that $\ddot{A}$ terms are negligible compared to $\dot{A}$ terms.

We determine the location of two attracting fixed points and one metastable saddle point from Eq. (2) for different values of parameters $F$ and $\sigma$ extracted from the experimental data. To calculate the separatrix, a set of points in state space that converge to the saddle point, we evolve the initial conditions lying close to this fixed point and along the negative-eigenvalue eigenvector [obtained by linearizing Eq. (2) around the saddle point], backwards in time according to Eq. (2). The curves generated by this procedure constitute the separatrix. To compare the theoretical calculations to the experimental data in Fig. 2, we scale computed amplitudes by the value of the critical amplitude, $a_{c}$, calculated from Eq. (2). We find good agreement between the experimental data and our theoretical calculations.

The separatrix observed in the experiment is blurred due to environmental noise affecting the system. These fluctuations perturb the initial state of the resonator and so cause uncertainty in the preparation of the initial state. For the states near the separatrix, switching the system to the bistable regime and forcing the initial state to project on either of the two stable states amplifies this jitter and results in the noisy separatrix in Fig. 2. Since the system is operated away from the bifurcation, the time scale on which noise affects the system is much slower than the ring-down time of the resonator and the dynamics can be analyzed in the small-noise approximation. We can then consider two scenarios for how external noise can affect the system, either additively or as parameter noise.

If noise in Eq. (2) for the initial state is additive, the evolution of the initial condition follows the deterministic path perturbed by small jitter until the system reaches the neighborhood of an attracting fixed point, corresponding to the initial state. The action of this noise is to perturb the system around the fixed point within a characteristic radius $\delta a$. If the additive noise in Eq. (1) is white with power spectral density $\gamma$, the effective temperature of the noise in the system described by Eq. (2) is $\frac{\gamma \alpha Q^{2}}{8 m^{2} \omega_{0}^{3}}$. From the equipartition theorem, $\delta a$ is given by $(\delta a)^{2} / a_{c}^{2}=\frac{3^{3 / 2}}{2} \frac{\gamma \alpha Q^{2}}{4 m^{2} \omega_{0}^{3}}$. We can estimate the uncertainty in the preparation of the initial amplitude from the observed blurring of the separatrix, $\delta a / a_{c}$, in Fig. 2 to be about $10 \%$. The force noise that would result in such blurring is $5 \mathrm{pN} / \sqrt{\mathrm{Hz}}$. For the Lorentz force due to the magnetomotive drive, this force noise translates to a voltage noise of $18 \mathrm{nV} / \sqrt{\mathrm{Hz}}$.

Alternatively, the noise can be present in the parameters of the system. The noise in the magnitude of the drive, $F$, results in the fluctuations of the position of the initial fixed point. Near the origin, where $a / a_{c}$ is small, the variation in the drive voltage $\delta V / V_{c}$ that would cause the $10 \%$ variation in amplitude is calculated from Eq. (2) to be $\delta V / V_{c}=$ $(\sqrt{3} / 2) a / a_{c}=8.7 \%$. (Farther away from the origin, fluc- 
tuations in the drive voltage have less effect on fluctuations in the amplitude due to nonlinear suppression.) Therefore, the observed blurring translates to fluctuations of $\delta V \approx$ $2.5 \mu \mathrm{V}$ for the measured critical drive, $V_{c}=28 \mu \mathrm{V}$. The relevant noise bandwidth for this resonator is $\pi f_{0} / Q=$ $24 \mathrm{kHz}$. The noise spectrum that would account for the $10 \%$ fluctuation in amplitude is then $16 \mathrm{nV} / \sqrt{\mathrm{Hz}}$. This result is consistent with the above estimate of fluctuations due to additive noise. The residual voltage noise from the initial-drive function generator and the rest of the drive circuit of about $5 \mu \mathrm{V} / \sqrt{\mathrm{Hz}}$ is attenuated by $51 \mathrm{dBm}$ by the rf switch and additional attenuators (not shown), and results in a voltage noise of $14 \mathrm{nV} / \sqrt{\mathrm{Hz}}$ at the sample. The drive-circuit noise therefore accounts for most of the observed fluctuations near the separatrix.

The presence of noise can also cause transitions from one fixed point to another [23,24]. We indeed observe the same noise-induced switching between two stable states as in References [5,25], where the noise from the drive source induces transitions of a resonator from one state to the other near the bifurcation points. By adding noise to the resonator drive and recording the statistics of the time it takes for the system to switch when it is near the bifurcation point, we find that the transition rate varies as $\exp \left(-E_{a} / \nu\right)$, where $\nu=k_{B} T_{\text {eff }}$ is the noise power and $E_{a}$ is the height of the energy barrier that the system needs to overcome for the transition to happen. The energy barrier depends on the distance to the bifurcation point $V_{b}: E_{a} \propto\left(V-V_{b}\right)^{\delta}$. We have measured the critical exponent $\delta$ to be $1.8 \pm 0.3$, which is close to the theoretically predicted value of $3 / 2$ in the region we operate in $[23,24,26]$. Measurement of transitions induced by noise in the bistable regime of a nanoscale resonator could enable a very sensitive experimental technique to detect mechanical fluctuations.

The experimental mapping of basins of attraction of a nanowire mechanical resonator presented here fills a large gap in our understanding of nonlinear dynamics of nanoscale systems. Since the nonlinear regime is readily accessible in nanoscale devices, the details of dynamical behavior are now increasingly important for proper engineering and analysis of these systems. The knowledge of basin dynamics and noise-induced transitions should also prove useful for precision measurement applications, such as nanomechanical bifurcation amplifiers [12], detection of transition to the quantum regime $[9,10]$, or a sensitive monitoring of intrinsic device noise processes.

We acknowledge partial support from the DARPA MTO/MGA (via DOI No. NBCH1050001). O. Kogan acknowledges support from the NSF Grant No. DMR0314069. We thank M. Barbic for building the nanowire fabrication setup and I. Bargatin and M. C. Cross for useful comments on the manuscript.
*Present address: Bosch Research and Technology Center, Palo Alto, CA 94304, USA.

${ }^{\dagger}$ Present address: Physics and Astronomy Dept., California State University at Northridge, Northridge, CA 913308268, USA.

${ }^{\ddagger}$ Present address: International Rectifier, El Segundo, CA 90245, USA.

${ }^{\S}$ To whom correspondence should be addressed. roukes@caltech.edu

[1] Y. T. Yang, C. Callegari, X. L. Feng, K. L. Ekinci, and M. L. Roukes, Nano Lett. 6, 583 (2006).

[2] D. Rugar, R. Budakian, H. J. Mamin, and B.W. Chui, Nature (London) 430, 329 (2004).

[3] H.W. C. Postma, I. Kozinsky, A. Husain, and M. L. Roukes, Appl. Phys. Lett. 86, 223105 (2005).

[4] B. Yurke, D. S. Greywall, A. N. Pargellis, and P. A. Busch, Phys. Rev. A 51, 4211 (1995).

[5] J. S. Aldridge and A. N. Cleland, Phys. Rev. Lett. 94, 156403 (2005).

[6] E. Buks and B. Yurke, Phys. Rev. E 74, 046619 (2006).

[7] I. Siddiqi, R. Vijay, F. Pierre, C. M. Wilson, M. Metcalfe, C. Rigetti, L. Frunzio, and M. H. Devoret, Phys. Rev. Lett. 93, 207002 (2004).

[8] M. D. LaHaye, O. Buu, B. Camarota, and K. C. Schwab, Science 304, 74 (2004).

[9] I. Katz, A. Retzker, R. Straub, and R. Lifshitz, Phys. Rev. Lett. 99, 040404 (2007).

[10] V. Peano and M. Thorwart, Phys. Rev. B 70, 235401 (2004).

[11] A. N. Cleland, New J. Phys. 7, 235 (2005).

[12] R. Karabalin, R. Lifshitz, S. Masmanidis, M. Cross, and M. L. Roukes (to be published).

[13] A. Husain, J. Hone, H. W. C. Postma, X. M. H. Huang, T. Drake, M. Barbic, A. Scherer, and M. L. Roukes, Appl. Phys. Lett. 83, 1240 (2003).

[14] A. Erbe, H. Kroemmer, A. Kraus, R. H. Blick, G. Corso, and K. Richter, Appl. Phys. Lett. 77, 3102 (2000).

[15] L. D. Landau and E. M. Lifshitz, Mechanics (Butterworth Heinemann, Oxford, 1981).

[16] J. P. Cusumano and B. W. Kimble, Nonlinear Dynamics 8, 213 (1995).

[17] L. N. Virgin, M. D. Todd, C. J. Begley, S. T. Trickey, and E. H. Dowell, Int. J. Bifurcation Chaos Appl. Sci. Eng. 8, 521 (1998).

[18] C. R. Martin, Science 266, 1961 (1994).

[19] A. N. Cleland and M. L. Roukes, Sens. Actuators A, Phys. 72, 256 (1999).

[20] K. Schwab, Appl. Phys. Lett. 80, 1276 (2002).

[21] A. H. Nayfeh and D. T. Mook, Nonlinear Oscillations (John Wiley \& Sons, New York, 1979).

[22] I. Kozinsky, H. W. C. Postma, I. Bargatin, and M. L. Roukes, Appl. Phys. Lett. 88, 253101 (2006).

[23] J. Kurkijarvi, Phys. Rev. B 6, 832 (1972).

[24] M. I. Dykman and M. A. Krivoglaz, Sov. Phys. JETP 50, 30 (1979).

[25] C. Stambaugh and H. B. Chan, Phys. Rev. B 73, 172302 (2006).

[26] O. Kogan and M. I. Dykman (to be published). 